\section{$\underset{\substack{\text { hommes } \\ \text { \& migrations }}}{ }$}

\section{Hommes \& migrations}

Revue française de référence sur les dynamiques

migratoires

$1301 \mid 2013$

Migrations et mondes ruraux

\title{
Entre captation et subjectivation
}

Les Travailleurs migrant-e-s et travailleurs délocalisé(e)s dans les abattoirs bretons

\section{Simona Tersigni et Nadine Souchard}

\section{(2) OpenEdition}

\section{Journals}

Édition électronique

URL : http://journals.openedition.org/hommesmigrations/1921

DOI : 10.4000/hommesmigrations. 1921

ISSN : 2262-3353

Éditeur

Musée national de l'histoire de l'immigration

Édition imprimée

Date de publication : 1 janvier 2013

Pagination : 89-96

ISBN : 978-2-919040-21-6

ISSN : $1142-852 X$

\section{Référence électronique}

Simona Tersigni et Nadine Souchard, «Entre captation et subjectivation », Hommes \& migrations [En ligne], 1301 | 2013, mis en ligne le 01 janvier 2015, consulté le 01 mai 2019. URL : http:// journals.openedition.org/hommesmigrations/1921; DOI : 10.4000/hommesmigrations.1921 


\title{
ENTRE CAPTATION ET SUBJECTIVATION
} LES TRAVAILLEURS MIGRANT-E-S ET TRAVAILLEURS
DÉLOCALISÉ-E-S DANS LES ABATTOIRS BRETONS

par SIMONA TERSIGNI, maître de conférences en sociologie à l'université Paris-Ouest-Nanterre (Sophiapol), et NADINE SOUCHARD, responsable de formation et de recherche au Collège coopératif de Bretagne (UMR CNRS ESO)

\author{
Dans les abattoirs bretons, une partie des travailleurs locaux \\ tend à être remplacée par des travailleurs migrants ou \\ détachés, originaires d'Europe de I'Est, du Maghreb ou d'Afrique \\ subsaharienne. Cette dynamique, difficilement perceptible, \\ a des effets concrets sur l'organisation du travail dans les \\ usines de transformation de la viande. Ces migrants y occupent \\ une place à part. Victimes de racisme, ils font les frais \\ de l'objectif de leur venue, réduire le coût de la main-d'œuvre \\ dans un secteur à faible valeur ajoutée.
}

\begin{abstract}
Sans vouloir confondre Bretagne et campagne, dans cet article, la référence à la ruralité renvoie au bassin de recrutement des ouvriers des abattoirs plus qu'à la localisation de ces entreprises situées, dans la plupart des cas, à proximité de petites communes de 10000 à 15000 habitants.
\end{abstract}

De plus en plus remarquée par les médias ${ }^{1}$, la présence de travailleurs migrants ou détachés en Bretagne, recrutés notamment dans l'industrie de la volaille et de la viande, échappe aisément à la comptabilité officielle de l'immigration. En effet, une partie considérable d'entre eux arrivent dans ce secteur de l'industrie agro-alimentaire (IAA) dans un contexte de prospection intensive de la part d'entreprises de prestation de services et d'intérim international, dont la libéralisation s'est effectuée en 2008². Sans vouloir confondre Bretagne ${ }^{3}$ et campagne, dans cet article, la référence à la ruralité renvoie au bassin de recrutement des ouvriers des abattoirs plus qu'à la localisation de ces entreprises situées, dans la plupart des cas, à proximité de petites communes de 10000 à 15000 habitants. 
Les ouvriers "bretons" des industries agro-alimentaires ${ }^{4}$ ont sacrifié au travail en abattoir la possibilité de préserver une localisation rurale, celle-ci impliquant pour eux un accès plus aisé à la propriété privée, une meilleure conciliation du temps de travail et des loisirs. En revanche, pour les ouvriers migrants ou détachés installés aujourd'hui dans des milieux ruraux bretons, on observe une dépendance de plus en plus grande à l'égard de l'employeur et, paradoxalement, une invisibilité sociale qui s'accompagne d'un

Malgré la multiplication

des abattoirs et de leur

zone de recrutement, dans

les campagnes bretonnes

touchées par le chômage,

ce réservoir de main-d'œuvre

non spécialisée a montré

ses limites. La substitution

des chômeurs "nationaux"

par des travailleurs

étrangers ne s'est pas faite

en quelques mois. faible accès à la sociabilité locale, voire un isolement. Ces ouvriers "allogènes", qui ne sont pas nécessairement salariés de l'entreprise dont ils contribuent fortement à accélérer la cadence, d'après plusieurs témoignages, résident dans des contextes ruraux, même quand les abattoirs se trouvent dans des communes comme Lamballe, Loudéac, Quimperlé ou Vitré. Ce qui avait auparavant été jugé par le patronat comme une "naturelle docilité des ruraux bretons" semble s'accompagner depuis la seconde moitié des années 2000 d'un changement rapide en termes de gestion internationale de la main-d'œuvre. Mais il n'est pas aisé de saisir ce changement. D'ailleurs, dans le processus de construction de la nation républicaine et jacobine, les Bretons ont réellement joué le rôle de "colonies de l'intérieur". Puis, plus récemment, plusieurs travaux, sur le mouvement breton des années $1930^{5}$ ou sur une famille juive roumaine assassinée en 1943 après avoir subi l'ex- clusion et la spoliation ${ }^{6}$, ont montré combien l'antisémitisme n’avait pas épargné la Bretagne. Tout en restant une terre de migrants et de navigateurs, elle a vu émerger en son sein des groupuscules d'extrême droite persuadés de la supériorité de la race celte tout comme l'application "autochtone" des mesures antisémites de l'occupant nazi.

Historiquement minorisés en France ${ }^{7}$, les Bretons sont à présent pris dans des contradictions pour lesquelles, à côté des mobilisations en soutien des sans-papiers déplacés depuis deux ans par la volonté du préfet de Rennes à Brest ${ }^{8}$, se fabriquent des pratiques salariales prises dans des logiques productivistes mondialisées. Celles-ci rendent extrêmement difficiles les tactiques des migrant(e)s, susceptibles de participer au déni ou à la dénégation $^{9}$ des majoritaires à leur égard. Aussi, souhaitons-nous montrer comment s'opère la localisation bretonne des rapports salariaux de l'industrie de la viande, qui se construisent d'une manière spécifique dans le cadre de "systèmes globaux ${ }^{10 "}$ propres à l'Union européenne.

\section{Chômeurs autochtones et travailleurs étrangers}

Au cours de deux différents moments d'enquête, entre début 2009 et fin de l'année 2010 ${ }^{11}$, et grâce à la collaboration de trois autres enquêteurs, nous avons progressivement saisi la portée d'un phénomène souvent considéré par les acteurs locaux comme étant de l'ordre de l'insaisissable : la substitution progressive, partielle et diversement appréhendable, des chômeurs "nationaux" par des travailleurs étrangers.

\footnotetext{
4. Les ouvriers des industries gérant la mise à mort et la transformation de la viande tout comme la production laitière ne sont pas à confondre avec les ouvriers agricoles, dont font état plusieurs articles dans ce même dossier. 5. Pierre-Jean Simon, "Racisme et antisémitisme dans le mouvement breton des années trente", in Pluriel, $n^{\circ} 18,1979$, pp. 29-38. 6. Marie-Noëlle Postic, Sur les traces d'une famille juive en Bretagne, Lorient, Coop Breizh, 2007. 7. Ronan Le Coadic, L'Identité bretonne, Rennes, Terres de Brumes/PUR, 1998; Pierre-Jean Simon, La Bretonnité. Une ethnicité problématique, Rennes, Terres de Brumes/PUR, 1999. 8. Voir http://www.galeresdebrest. $\mathrm{fr} / 2012 / 12 / 21 / a p r e s-2$-ans-la-situation-des-migrants-a-brest-est-toujours-la-meme-a-la-rue-et-repression/

9. Didier Fassin, Éric Fassin, "Introduction", in De la question sociale à la question raciale? Représenter la société française, Paris, La Découverte, 2006. 10. Jonathan Friedman, “Concretizing the continuity argument in global systems analysis", in Jonathan Friedman et al., World System History. The Science of Long Term Change, Londres, Routledge, 2000. À l'instar de cet anthropologue, il s'agit de mettre l'accent sur des processus systémiques qui relient les différentes dimensions locales du monde et même leurs processus de formation, en tant que lieux plus ou moins circonscrits en connexion avec un phénomène étudié, en l'occurrence la présence dissimulée d'ouvriers étrangers détachés, intérimaires ou recrutés au sein des abattoirs bretons.
} 
Malgré la multiplication des abattoirs et de leur zone de recrutement, dans les campagnes bretonnes touchées par le chômage, ce réservoir de maind'œuvre non spécialisée a montré ses limites. La substitution des chômeurs "nationaux" par des travailleurs étrangers ne s'est pas faite en quelques mois. Ainsi, des entreprises comme Kermené ont recruté au début des années 2000 des chômeurs de la région de Valencienne après que ses entreprises de Collinée (abattoirs Gilles à l'époque) avaient eu recours à des Maliens depuis les années 1970, comme l'ont montré Angelina Étiemble et Anne Morillon $^{12}$.

Notre hypothèse initiale se trouvant infirmée, nous avons également dû déconstruire des discours stéréotypés tels que celui du président de l'Association malienne de Collinée (déjà rencontré et très médiatique). D’autre part, alors que nous voulions prendre de la distance par rapport aux récits de la presse locale ${ }^{13}$ pointant les expulsions et les mobilisations citoyennes en soutien de salariés sans titre de séjour, notre recherche n'avait de cesse de prendre des allures d'enquête journalistique. De toute manière, la présence de travailleurs migrants ou détachés continuait de s'imposer, garantissant tantôt une augmentation de la cadence tantôt la réalisation d'un travail qu'une partie des chômeurs "autochtones" ne souhaitait plus faire. Ainsi, nous avons essayé de comprendre en quoi et si des formes de 3 D jobs (Dirty, Demanding, Dangerous) dans les abattoirs bretons pouvaient finir par concentrer l'essentiel des activités proposées à des travailleurs étrangers, de manière à en--tremêler tertiarisation, flexibilité et informalisation du travail dans le cadre de tâches indicibles, associées à la transformation de l'animal en substance consommable. Il s'agissait d'appréhender dans quelles conditions et avec quels effets ce refus des Français socialement minorisés (les ouvriers bretons, les chômeurs bretons, les chômeurs du Nord) d'assister ou de participer à la souffrance de la mise à mort des animaux - dont pourtant Noélie Vialles ${ }^{14}$ a finement étudié une dissociation en chaînes - s'accompagnait d'un recours à des "travailleurs détachés" en tant que "principale modalité de recrutement de migrants". Selon les interlocuteurs de notre première recherche, ce processus était difficilement saisissable, voire marginal et, in fine, dénué d'intérêt. Il pouvait, par ailLes industries des viandes ont précocement installé en leur sein des formes de dérégulation, touchant précisément les segments les plus qualifiés. leurs, être une source d'inquiétude puisqu'il participait d'une segmentation des collectifs de travail du fait de l'importance prise par l'intérim national et international, ainsi que par les entreprises de tâcheronnat et de prestations de services internationales. Alors que le modèle du tâcheronnat paraissait s'essouffler, les avantages comparatifs de cette forme de travail s'estompant et les candidats locaux étant objectivement moins nombreux, ces entreprises ont expérimenté le recours à une main-d'œuvre étrangère depuis la fin des années 1990. Ainsi, des Vietnamiens ont été recrutés par Euroviande, dans le cadre d'accords bilatéraux sous le gouvernement de Jospin, et ensuite mis en disposition ou recrutés chez Bigard et à la Société vitréenne d'abattage (SVA).

Les industries des viandes ont précocement installé en leur sein des formes de dérégulation, touchant précisément les segments les plus qualifiés. L'industrialisation de l'abattage et de la découpe dans les années 1980, outre le fait qu'elle a considérablement modifié les conditions de la mise à mort animale ${ }^{15}$, s'est traduite par une intensifica- 
tion et une déqualification progressives du travail. Traditionnellement réalisés par des bouchers opérant comme des travailleurs indépendants et de ce fait payés à la tâche, la mise à mort, le désossage et le parage de la viande constituent encore les étapes essentielles d'un processus productif aujourd'hui tourné vers une production de masse. Or les entreprises sous-traitantes (les "tâcherons") ont contribué en partie à pallier le déficit de main-d’œuvre de cette industrie. Toutefois, la référence persistante au "tâcheronnat" a pu occulter la situation salariale des "tâcherons" et fait davantage référence à une idéologie du travail indépendant, au détriment d'une inscription claire dans un statut salarial. Sans pouvoir nous étendre sur le sujet, la place à part des tâcherons dans les usines en termes de tapis de travail et de salaire proportionnel au rendement est un fonctionnement qui s'est reproduit dans le cadre de nouvelles formes d'intérim et de prestations de services internationales ${ }^{16}$. Ainsi, en continuité avec des pratiques antérieures ${ }^{17}$, se reconstitue aujourd'hui une main-d'œuvre rompue à la discipline usinière et travaillant dans des conditions financières défiant toute concurrence.

\section{Entre déni et dénégation: postures de quelques professionnels en lien avec les travailleurs étrangers}

La substitution ${ }^{18}$ d'une partie des travailleurs locaux par des étrangers est relativement récente dans les abattoirs étudiés en Bretagne et n'est pas aussi massive que dans d'autres secteurs (agriculture, bâtiment, hôtellerie, notamment). Toutefois, cette substitution s'est réalisée suivant différentes formes qui ont contribué à rendre invisibles ou opaques les conditions de recrutement de la main- d'œuvre étrangère. Seul un document produit en 2008 dans le cadre des contributions du Comité de développement du pays de Rennes (Codespar) est assez éclairant sur les orientations qui se dessinent, face aux difficultés d'automatisation du cycle productif du fait du caractère vivant du produit, mais aussi, pour l'industrie des viandes, parce qu'elle s'apparente davantage à une industrie de série. Si la substitution homme/machine reste difficile à réaliser, l'importance des volumes traités dans les abattoirs bretons a orienté les entreprises vers des productions à faible valeur ajoutée. Ces entreprises et coopératives dégagent l'essentiel de leur marge grâce au tonnage de production animale transformée. Si le coût de la main-d'œuvre pèse singulièrement sur le chiffre d'affaires de ces entreprises ${ }^{19}$, selon l'Association pour le développement de l'emploi et de la formation dans les industries agroalimentaires de Bretagne (Adefia ${ }^{20}$ ) "l'export de viande française à prix fort dans les pays de l'Europe de l'Est peut ne pas être pérenne et le rapport pourrait s'inverser à terme [avec] un scénario de disparition pure et simple de cette activité, les entreprises de transformation".

Ce document est, à notre connaissance, le seul où la profession ait reconnu publiquement s'orienter vers le recours à des populations étrangères peu qualifiées, non pas officiellement pour réduire le coût de la main-d'œuvre, mais parce que le modèle de recrutement traditionnel qui privilégie "la recherche d'une force de travail plus que des compétences à proprement parler ${ }^{21 "}$ continuerait de prévaloir dans la mise à mort des animaux dont les volumes n'ont cessé de croître ces dernières années. Aussi, les plus importantes entreprises bretonnes travaillent-elles régulièrement avec différentes agences intérimaires pour satisfaire leurs besoins de main-d'œuvre précaire, tout en continuant à organiser en leur sein l'accueil de demandeurs d'emplois pour susciter des

16. Ces entreprises européennes, qui mènent un travail de prospection assidu auprès des DRH de l'agro-alimentaire et sur leurs sites Internet, évoquent assez crûment les avantages des services qu'elles proposent. 17. Dans les abattoirs, il était courant d'avoir des salariés soumis à des rythmes de travail plus intenses, sur des tapis séparés et qui, de ce fait, n'étaient pas perçus comme faisant partie du collectif de travail. 18. Jusqu'à la seconde moitié des années 1980, le mot “substitution" a été employé en sociologie des migrations pour désigner dans les usines la requalification de certains postes occupés par les migrants et leur attribution à des ouvriers nationaux. 19. Selon l'Adefia, le coût relatif de la main-d'œuvre représenterait 73 \% de la valeur ajoutée en Bretagne contre 55 \% en France. 20. Association d'industries agro-alimentaires bretonnes (réseau de collaboration et d'innovation sociale). 21. Contributions du Codespar, 2008. 
candidatures. Face à l'ampleur des troubles musculo-squelettiques dans ce secteur (les TMS viennent d'être reconnus comme une maladie professionnelle ${ }^{22}$, la déqualification et la féminisation de certains postes ont été relevées par les DRH des coopératives et des entreprises. Ces dirigeants sont toutefois dans le déni ou expriment une reconnaissance limitée à l'égard du recrutement des travailleurs migrants ou détachés. Il s'agissait pour eux de dissimuler une lutte menée à l'échelle européenne en tant que représentants de la Fédération française des viandes avec la même fédération outreRhin, cette dernière ayant depuis longtemps fait le choix de recruter des travailleurs originaires des anciens pays du bloc soviétique dans un contexte allemand dépourvu de Smic. Ces attitudes de déni et de dénégation qui prévalaient souvent chez les DRH bretons prenaient appui sur une externalisation partielle des recrutements. Parmi les ouvriers, les salariés et l'ensemble des délégués syndicaux, ces interrogations étaient aussi alimentées par l'absence de visibilité de cette main-d'œuvre et sa découverte souvent hasardeuse au sein des établissements, suscitant tantôt incompréhension, tantôt spéculations. La mobilisation syndicale sur le sujet, par exemple, n'était pas forcément proportionnelle au nombre de personnes concernées. Nous avons été parfois surprises par le décalage entre l'intérêt syndical et la visibilité du phénomène, mais l'inverse est aussi identifiable! De plus, un décalage important se creusait entre le travail de fond mené à Bruxelles à l'échelle d'une fédération agro-alimentaire - en l'occurrence celle de la CFDT - et le maillage quotidien de ces questions par des élus syndicaux souvent déconnectés du terrain ou placés dans l'impossibilité de comprendre ce qui était en train de se passer. Ainsi, nos interlocuteurs syndicaux ont eu beaucoup de mal à chiffrer les effectifs de travailleurs migrants. Les syndicalistes réclamaient d'ailleurs régulièrement ces données auprès de leurs établissements. Seule une délégation syndicale, celle de la CGT, a explicitement refusé de nous recevoir en indiquant qu'à sa connaissance seules trente personnes étaient dans ce cas sur un effectif qui approchait les 2000 salariés. Pourtant, un délégué CFDT de cette même entreprise estimait le processus suffisamment avancé pour s'impliquer dans une réflexion confédérale sur les migrations et la ruralité.

L'inspection du travail paraissait un peu plus nuancée, même si elle était prise entre des orientations citoyennes, les problèmes endémiques spécifiquement liés aux industries agro-alimentaires et l'attitude consistant à déclarer que "rien ne remonte". Les inspecteurs de la direction du Travail finissaient au mieux par s'attacher à des définitions juridiques de la discrimination, conduisant à légitimer des décisions qui soumettaient les étrangers à un statut dérogatoire par rapport aux travailleurs nationaux, tout en restant dans l'application de la loi. D'autres inspecteurs semblaient avant tout préoccupés par l'urgence de fournir

Si la substitution homme/machine reste difficile à réaliser, l'importance des volumes traités dans les abattoirs bretons a orienté les entreprises vers des productions à faible valeur ajoutée. un flux de main-d'œuvre suffisant pour faire tourner des établissements pensés pour traiter des volumes importants. Il en résultait une relative invisibilité des travailleurs migrants originaires de l'Europe de l'Est sur les sites de production, ce qui renvoyait à une invisibilisation administrative tout autant qu'opérationnelle. Quant aux dirigeants, pressés par les délégués syndicaux de donner des chiffres précis, ils se réfugiaient le plus souvent derrière l'extranéité des entreprises ou leur caractère de sous-traitant.

\section{Dérives asservissantes et subjectivation}

Invisibilisés par les pratiques de recrutement pour certains, pour d'autres par l'absence de discours patronal et syndical les concernant, ces travailleurs migrants l'étaient également par l'intériorisation d'un pacte unilatéral d'invisibilisation sur les lieux 
de travail, de manière analogue à ce qu'ont observé Alain Morice et Swanie Potot ${ }^{23}$. Néanmoins, lorsque les entretiens ont pu avoir lieu (nombreux refus ou annulations de dernière minute), les discours sont restés souvent extrêmement contrôlés, frisant parfois la contradiction. Ainsi, Marek (Polonais en CDI) a oscillé entre "Ils placent les Polonais aux postes où les Fran-

Ces travailleurs migrants

se sont rarement autorisés

à laisser transparaître

les problèmes rencontrés

sur les lieux de travail,

sauf quand ils avaient quitté

l'entreprise au moment

des entretiens. çais ne veulent pas être, aux tâches les plus ingrates" et "Je suis content de mon poste". Ces travailleurs migrants se sont rarement autorisés à laisser transparaittre les problèmes rencontrés sur les lieux de travail, sauf quand ils avaient quitté l'entreprise au moment des entretiens. En revanche, ils ont manifesté une idéalisation de leurs conditions de travail qui ont parfois pu être interrogées grâce à des situations d'entretiens diversifiées par la présence d'une tierce personne.

Au moment d'une "politique d'immigration choisie" réalisée sous la présidence de Nicolas Sarko$\mathrm{zy}^{24}$, nous avons rencontré des salariés des pays de l'Est ${ }^{25}$, théoriquement en situation de détachement, Roumains, Polonais, Ukrainiens ; des "aventuriers" qui, en itinérance souvent depuis l'Afrique, tentaient de régulariser leur situation par l'obtention d'un contrat de travail ; des Marocains installés en France depuis quelques années avant que ne se durcissent les conditions d'entrée sur le territoire français ; des demandeurs d'asile déboutés de leurs droits, mais qui avaient réussi à obtenir un titre de séjour avec la mention "travail" du fait de leur recrutement dans des métiers réputés sous tension ${ }^{26}$.
La reconstruction des trajectoires individuelles de carrière des migrants et des travailleurs détachés ${ }^{27}$ a permis de mieux comprendre que les syndicalistes avaient du mal à prendre en compte cette opulation dans leur action, ou qu'ils ne le souhaitaient pas. Il ne pouvait pas y avoir de réel consensus syndical sur un sujet susceptible d'alimenter autant de sentiments ambivalents chez les salariés "autochtones". Les propositions d'embauche en CDD ou CDI des travailleurs migrants et des salariés de prestataires dans des emplois statutaires étaient marquées du sceau de l'ambivalence, leur recrutement requérant un don de soi, lequel passait par l'acceptation de souffrances corporelles et d'humiliations racistes. Certains des travailleurs migrants rencontrés correspondaient à ce qu'Anaïk Pian considère comme des "aventuriers à mobilité territoriale marquée ${ }^{28 ”}$, puisqu'une partie d'entre eux parvenaient à intégrer, en région parisienne, d'autres secteurs de production considérés comme plus alléchants, l'automobile, par exemple. Toutefois, ces hommes comme ces femmes étaient dans l'ensemble captés par l'industrie des viandes, et l'inscription dans le CDI, qui peut apparaître comme une sécurité (et qui a fait l'objet de luttes syndicales), devenait pour eux une manière de sacrifier leurs carrières migratoires puisqu'ils ne se destinaient pas, a priori, à de tels emplois, notamment si on considère leurs qualifications ou projets migratoires.

Aussi, le collectif de travail était-il un espace problématique en soi et ils avaient de bonnes raisons pour ne pas aborder le racisme dont ils étaient victimes au quotidien. Somme toute, nous avons été progressivement frappées par une négation initiale du racisme, puis par sa naturalisation (et son internationalisation), par exemple : “Ça existe

\footnotetext{
23. Alain Morice, Swanie Potot (dir.), De l'ouvrier immigré au travailleur sans papiers. Les étrangers dans la modernisation du salariat, Paris, Karthala, 2010, pp. 307-316. 24. Sous la présidence de François Hollande, la circulaire du ministre de l'Intérieur Manuel Valls (28 novembre 2012) a renforcé ce dispositif en soumettant l'obtention d'un titre de séjour à un contrat ou à une promesse d'embauche, comme cela s'est déjà produit dans des nouveaux pays d'immigration tels que l'Italie quelques années auparavant. 25. Ils relevaient d'une vingtaine d'entreprises et coopératives, de cinq enseignes de l'intérim national, de cinq prestataires de services et, enfin, de quatre entreprises pratiquant l'intérim ou la prestation de services à l'échelle européenne. 26. Daté du 18 juin 2010, un communiqué de presse du ministère de l'Immigration, de l'Intégration, de l'Identité nationale et du Développement solidaire rend compte d'une rencontre avec plusieurs syndicats (CGT, CFDT, FSU, Solidaires et Unsa) afin de détecter les difficultés d'application dans certains secteurs professionnels, dont l'intérim. 27. Nos 17 interlocuteurs échappent tous et toutes aux stéréotypes véhiculés dans les IAA par les délégués syndicaux, les DRH ou les inspecteurs du travail. 28. Anaïk Pian, Aux nouvelles frontières de l'Europe, Paris, La Dispute, 2009.
} 
partout, ça existe aussi en Afrique", puis par son euphémisation suivant le mode "J'aime le sang" (Joseph, migrant congolais), laissant entendre la violence répandue et implicite dans ce contexte professionnel...

\section{Des logiques racisantes sous-jacentes}

Nous avons distingué quatre postures à l'égard du racisme chez ces travailleurs et travailleuses. Elles reviennent à nier le racisme, à en fairele constat sous des modalités euphémisées, à se convaincre continuellement de pouvoir faire avec, ou alors à lutter contre. Certains de nos interlocuteurs ou interlocutrices pouvaient, en fonction des situations, assumer l'une où l'autre, alors qu'elles pourraient nous sembler complètement contradictoires.

La posture la moins répandue revenait à lutter contreleracisme. Unmigrantcentrafricain,Étienne, racontait notamment que les ouvriers bretons de son usine savaient pousser à bout les migrants à l'égard desquels ils manifestaient clairement de l'hostilité : "Si ta tête ne plaît pas, on te pousse à bout pour que tu partes tout seul." En tant que délégué syndical dans son entreprise, il lui était arrivé de rendre visite aux salariés : une fois, un ouvrier avait fait semblant de ne pas le voir, voulant ignorer sa responsabilité syndicale, une autre fois, un autre ouvrier avait carrément refusé de lui serrer la main... Rarement a été mis en avant un savoir-faire en matière d'autodéfense en situation de racisme. Joseph, Congolais torturé avant de quitter son pays, a plutôt mis l'accent sur le nombre de migrants ayant changé d'entreprise et de lieu de vie, dès lors qu'ils ne supportaient plus les conflits et les injures. Seulement quelques-uns réagissaient verbalement ou physiquement aux déclarations et aux actes racistes, sans pouvoir pour autant contrer les logiques racisantes qu'ils subissaient, comme l'a expliqué Aziz, migrant marocain.
Même s'il n'y avait pas de segmentation du travail sur des fondements clairement raciaux, des expérimentations étaient bien à l'œuvre : les Vietnamiens, les Maliens, les Roumains occupaient bien des tapis à part... Qui plus est, des préjugés racistes, souvent rapportés par des tiers, nous informent des discours tenus sur les mises à l'épreuve dans des espaces usiniers où il s'agit de tenir les cadences, là où il n'y a pas de polyvalence. De nombreux travailleurs migrants étaient tenus à l'écart pendant les pauses en dépit de leurs efforts. Des mises à l'épreuve existaient également au sortir de l'usine dans les espaces de sociabilité où il s'agissait de boire un verre avec des collègues, ce qui s'imposait comme la condition de l'acceptation dans le collectif de travail, alors que les croyances religieuses le prohibent pour beaucoup...

Accepter l'inacceptable était donc le lot commun de nombreux travailleurs. Nous en avons constaté plusieurs fois les conséquences, comme une ouvrière arménienne, Natalia, contrainte de faire le trajet en
Aussi, le collectif de travail était-il un espace problématique en soi et ils avaient de bonnes raisons pour ne pas aborder le racisme dont ils étaient victimes au quotidien. voiture plusieurs fois par jour, en pleurs, avec sa fille. La trajectoire d'Aziz a montré comment l'aspiration à la mobilité (ne serait-ce que par la tentative d'acquisition d'un permis de conduire français) demeurait la principale modalité de résistance à la captation et à l'exploitation. Cette aspiration avait pourtant du mal à se concrétiser, certains interlocuteurs laissant entrevoir l'enfermement matériel et symbolique dans lequel ils étaient pris. De ce point de vue, le fait que la plupart de ces travailleurs résident à proximité de leur usine suggère que, derrière l'assignation laborieuse, se cache une forme d'assignation à résidence. Le logement collectif et contrôlé pour les travailleurs détachés, tout comme les logements sociaux ou patronaux (la différence n'est d'ailleurs pas toujours très claire chez nos interlocuteurs), installent une dépendance par le logement, stimulant un sentiment ambivalent de nécessaire gratitude. 


\section{Conclusion}

La Bretagne a été et reste animée par des réflexions et des perspectives de type régionaliste qui compliquent la prise de conscience d'une réalité migratoire. Il en découle fréquemment la prédominance de références à un développement économique endogène, pensé comme une modalité régionale équilibrée et comme un frein à l'émigration, même s'il y a une conscience et une reconnaissance des emplois faiblement qualifiés et des problèmes environnementaux. La région Bretagne tout comme les représentants de l'industrie agro-alimentaire ne se sont pas clairement positionnés sur l'expérimentation de ce qu'il convient d'appeler à la suite d'Emmanuel Terray "le travail délocalisé sur place ${ }^{29}$ ”, alors même que l'accélération du processus de concentration économique dans ce secteur induit de nouvelles pratiques managériales, également observables dans d'autres pays européens (Irlande, Pays-Bas, Allemagne...). En continuité avec des formes de "servitude vo- lontaire" dont les travailleurs du monde rural breton ont été "naturellement" jugés porteurs par le management français, ces nouvelles formes d'exploitation et de corvéabilité sont bien ancrées dans un maillage liant entre eux plusieurs pays ressortissants de l'UE, venant de l'intégrer ou candidats à ce regroupement politique. La réalité laborieuse qui conditionne la présence de ces "allogènes" sur les territoires ruraux bretons nous semble renforcer concrètement l'ethnicisation et la racisation des rapports sociaux, dont la lecture ne s'impose pourtant pas. La multiplication de différents statuts contractuels d'ouvriers étrangers se réalise dans le cadre d'une banalisation de l'ordre racial dont les auteurs d'autres publications relativement récentes sur la Bretagne ${ }^{30}$ n'avaient pas encore pris conscience. Or le caractère diffus, étendu et diversifié d'un recours non massif aux travailleurs migrants et étrangers dans les abattoirs bretons interroge tout à la fois le don de soi qu'il requiert de la part des travailleurs étrangers des industries des viandes et renouvelle, en milieu rural, l'ambiguiité syndicale française sur le thème de la "concurrence déloyale" des étrangers... 